\title{
Editorial
}

\section{Tempos de Renovação}

\author{
Valeu a pena? Tudo vale a pena \\ Se a alma não é pequena. \\ Quem quere passar além do Bojador \\ Tem que passar além da dor \\ Deus ao mar o perigo e o abysmo deu, \\ Mas nelle é que espelhou o céu. \\ Fernando Pessoa, \\ Mensagem
}

O fim de um ano sempre enseja uma retrospectiva, uma avaliação crítica e uma prospectiva para o ano vindouro. Nesse clima encerramos mais um volume de nossa revista. O ano de 2006 foi, para a atual gestão da revista Psicologia: Teoria e Pesquisa, um ano preparatório para grandes mudanças. Embora não se tenham feito sentir pelo leitor, tais mudanças vêm se operando nos bastidores de nossa revista e se prenunciam como grandes desafios para o ano de 2007. Refiro-me principalmente a duas grandes empreitadas: passar a operar em bases eletrônicas (Sistema Eletrônico de Editoração de Revistas - SEER) e passar a ter periodicidade trimestral.

Acompanhando tais mudanças, a revista também renovou seu estatuto interno, seguindo os rumos institucionais na esteira da reforma universitária. Além disto, a revista vem passando por uma reforma de seu espaço físico, que como é de costume, invariavelmente provoca alguns inconvenientes e transtornos, ainda que com um nobre propósito. Diante desse turbilhão de eventos, queremos agradecer a compreensão de nossos parceiros, usuários, assinantes, autores, conselheiros e pareceristas e nos desculpar pelos percalços decorrentes de tão delicado momento. Deposito grande esperança nas mudanças prenunciadas e acredito que a agilidade na tramitação eletrônica irá diminuir sensivelmente as intercorrências de natureza semelhante.

Coincidentemente a tantas modificações estruturais, nosso corpo editorial de Conselheiros nacionais e internacionais também se vê renovado em parte de seus membros neste último número, findo o prazo previsto pelo Estatuto da revista. Aos conselheiros Áderson Luiz Costa Júnior, Ana Cecília de Souza Bastos, Bill Fullford, Hartmut Günther, Jaan Vasiner, Jairo Eduardo Borges-Andrade, Maria Fátima Olivier Sudbrack, Maria Helena Leite Hunziker e Regina Helena Freitas Campos que encerram suas missões, nossos sinceros agradecimentos pela incansável tarefa de defender o caráter da revista como órgão representativo de todas as tendências da Psicologia do país, zelar pela alto nível científico e informativo, emitir suas apreciações críticas quando solicitados e por sua valiosa colaboração nos interesses de nosso periódico. Nossas boas vindas aos novos conselheiros Adriana Wagner, Alberto Rosa, André Berten, Gerson Aparecido Yukio Tomanari, Lucia Rabello de Castro, Maria Izabel Tafuri, Mário César Ferreira, Tereza Cristina Cavalcanti Ferreira de Araújo e Wanderley Codo.

O leitor pode apreciar neste número contribuições de autores de âmbito nacional e internacional nos diferentes domínios da Psicologia. Quanto à relação mãe-criança, no artigo de Ana Villas Boas, Olga Rodrigues e Midori Yamada apresenta-se um estudo de caso no qual destacase a importância de intervenções que favoreçam tal relação para desenvolver habilidades comunicativas da criança com deficiência auditiva. Os transtornos de atenção no desenvolvimento infantil são avaliados no artigo de Rodrigo Graeff e Cícero Vaz, no qual buscou-se investigar os aspectos de personalidade de crianças com Transtorno de Déficit de Atenção e Hiperatividade por meio do Rorschach. Quanto às relações família-escola, o artigo de Sandra Conte de Almeida, Maria Cristina dos Santos e Tânia Rossi focaliza as representações sociais de professores do ensino fundamental acerca da violência intrafamiliar.

Neste número apresenta-se uma série de contribuições da área de psicometria: Solange Wechsler e Patricia Schelini investigam a validade de construto da Bateria WoodcockJohnson III para avaliar as habilidades cognitivas de brasileiros. Evely Boruchovitch, Acácia dos Santos, Elis da Costa, Edna Neves, Miriam Cruvinel, Ricardo Primi, Sueli Edi Guimarães apresentam um estudo preliminar de suas propriedades psicométricas de uma escala para avaliar as estratégias de aprendizagem de alunos do ensino fundamental. José Bueno, Priscilla Santana, Juliana Zerbini e Thales Ramalho estudam as propriedades psicométricas de um instrumento para avaliação da inteligência emocional de estudantes universitários traduzido do inglês para o português. Eliane Seidl e Bartholomeu Tróccoli investigam a estrutura fatorial e as propriedades psicométricas da Escala de Suporte Social para Pessoas Vivendo com HIV/aids, elaborada com base em itens de uma escala canadense.

A avaliação da percepção visual é tratada no artigo de Cesar Galera, Mikael Cavallet, Michael von Grünau e Afroditi Panagopoulos, no qual investiga-se as características do foco atentivo por meio do efeito de dicas locais múltiplas e globais, múltipla e unitária, sobre o desempenho em uma tarefa de busca visual. Por sua vez, Valtenice França, Natanael Santos e Liana Mendes utilizam o método psicofísico da escolha forçada para mensurar a função de sensibilidade ao contraste para frequiências espaciais. A relação entre características de personalidade e a suscetibilidade a falsas memórias é tratada no artigo de Luciana de Ávila e Lilian Stein.

$\mathrm{Na}$ área de análise experimental do comportamento, Maria Helena Hunziker, Marcos Yamada, Fernando Manfré e Érika de Azevedo apresentam um estudo para verificar se a experiência prévia com eventos aversivos incontroláveis interfere na aprendizagem da variabilidade ou da repetição operantes.

$\mathrm{Na}$ área da História da Psicologia, Renato Kinouchi discute em seu artigo o papel do darwinismo na psicologia jamesiana, a partir de uma análise histórico-conceitual.

Um estudo de caso clínico com base na análise fenomenológica é apresentado no artigo de Rafael dos 
Santos, Célia de Moraes e Adriano Holanda, no qual os autores propõem uma avaliação do papel da ayahuasca - de uso em contexto religioso - na redução do consumo abusivo de psicoativos.

Por fim, Rosani Gambatto e André Luiz da Silva apresentam uma resenha sobre o livro "Sem filhos: a mulher singular no plural" de Luci Helena Baraldo Mansur, no qual o tema da não-maternidade - fenômeno freqüente e pouco investigado nos meios acadêmicos - é discutido.

Como de costume, por ser o último número do ano, consta neste exemplar, a lista de pareceristas ad hoc de 2006 e o índice dos autores e assuntos publicados ao longo do volume 22.

Em tempos de renovação, enquanto editora da revista Psicologia: Teoria e Pesquisa reitero o compromisso de manter os padrões exigidos na área de editoração científica em Psicologia, norteada pelo rigor e pela ética na condução de minhas atribuições.

Aproveito, ainda, os tempos de renovação para desejar votos de um futuro melhor para todos. Desejo à editoração científica brasileira que as políticas de fomento da pesquisa científica e tecnológica, ao avaliarem os periódicos científicos, voltem seus olhares para as especificidades das áreas de conhecimento, sejam mais sensíveis às necessidades de investimento financeiro para garantir o progresso da ciência em nosso país, que não se rendam aos valores mercantilistas subjacentes à atual tendência de incremento na produção dos pesquisadores de instituições de ensino superior e que valorizem (como, enfim, tem-se feito ultimamente) o trabalho de editores, revisores, pareceristas ad hoc e membros de conselhos editoriais como um produto científico relevante.

Desejo a todos um Natal cheio de luz e um Ano Novo de brilhantes renovações!

$\mathrm{Ah}$ ! Em tempo de renovações, aproveitem para renovar suas assinaturas...

\section{Referência}

Pessoa, F. (1941). Mensagem. Lisboa: Agencia Geral das Colonias.

\section{Conselho Editorial (2004 - 2006)}

Adriana Wagner (PUC-RS)

Ana Cristina Costa de Figueiredo (UFRJ)

Ângela Maria de Oliveira Almeida (UnB)

Antonio Pedro de Mello Cruz (UnB)

Domingos Sávio Coelho (UnB)

Emmanuel Zagury Tourinho (UFPA)

Eveline Maria Leal Assmar (UGF)

Gerson Aparecido Yukio Tomanari (USP)

Leandro de Lajonquière (USP)

Lúcia Rabello de Castro (UFRJ)

Maria Izabel Tafuri (UnB)

Mário César Ferreira (UnB)

Tereza Cristina Cavalcanti Ferreira de Araújo (UnB)

Terezinha de Camargo Viana (UnB)

Valdiney Veloso Gouveia (UFPB)

Wanderley Codo (UnB) 


\section{Psicologia: Teoria e Pesquisa \\ Consultores Ad Hoc - 2006}

Acácia Aparecida Angeli dos Santos

(Universidade São Francisco)

Ada Clarice Gastaldi

(Universidade de São Paulo)

Áderson Luiz Costa Júnior

(Universidade de Brasília)

Adriana Wagner

(Pontifícia Universidade Católica do Rio Grande do Sul)

Adriano Furtado Holanda

(Universidade de Brasília)

Adrian Oscar Dongo Montoya

(Universidade Estadual Paulista Júlio de Mesquita Filho)

Alessandra da Rocha Arrais

(Universidade Católica de Brasília)

Alessandra Rocha de Albuquerque

(Universidade Católica de Brasília)

Alessandra Gotuzo Seabra Capovilla

(Universidade São Francisco - Itatiba)

Alexandre Saadeh

(Pontifícia Universidade Católica de São Paulo)

Alina Galvão Spinillo

(Universidade Federal de Pernambuco)

Amauri Gouveia Jr

(Universidade Estadual Paulista Júlio de Mesquita Filho)

Amer Cavalheiro Hamdan

(Universidade Federal do Paraná)

Ana Cecília de Sousa Bittencourt Bastos

(Universidade Federal da Bahia)

Ana Cristina Costa de Figueiredo

(Universidade Federal do Rio de Janeiro)

Ana Lúcia Galinkin

(Universidade de Brasília)

Anamaria Silva Neves

(Universidade Federal de Uberlândia)

Ana Regina Noto

(Universidade Federal de São Paulo)

Ana Teresa de Abreu Ramos Cerqueira

(Universidade Estadual Paulista Júlio de Mesquita Filho)

Andréia Schmidt

(Centro Universitário Positivo)

Ângela Maria Regis Cavalcanti Brasil

(Universidade Presbiteriana Mackenzie)

Ângela Maria de Oliveira Almeida

(Universidade de Brasília)

Ângela Maria Cristina Uchoa de Abreu Branco

(Universidade de Brasília)

Anna Carolina Lo Bianco Clementino

(Universidade Federal do Rio de Janeiro)

Antonio Bento de Moraes

(Universidade Estadual de Campinas)

Antonio Egídio Nardi

(Universidade Federal do Rio de Janeiro)

Antonio Pedro de Mello Cruz.

(Universidade de Brasília)

Arthur Arruda Leal Ferreira

(Universidade Federal do Rio de Janeiro)

Bader Burihan Sawaia

(Pontifícia Universidade Católica de São Paulo)

Balduíno Antonio Andreolo

(Centro Universitário La Salle)

Beatriz Carmem Warth Raymann

(Universidade Luterana do Brasil)

Beatriz Senoi Ilari

(Universidade Federal do Paraná)

Bellkiss Wilma Romano
(Universidade de São Paulo)

Bernardo Jablonski

(Pontifícia Universidade Católica do Rio de Janeiro)

Bernard Pimentel Range

(Universidade Federal do Rio de Janeiro)

Carlos Henrique Sancineto

(Universidade São Francisco)

Cássia Maria Ramalho

(Centro Universitário de Brasília)

Cássia Regina Rodrigues Varga

(Faculdade de Medicina de Marília)

Cecília Maria Bouças Coimbra

(Universidade Federal Fluminense)

Célia Maria Lana da Costa Zannon

(Universidade de Brasília)

Célia Regina Vieira de Souza Leite

(Centro Universitário Moura Lacerda)

Celso Pereira de Sá

(Universidade do Estado do Rio de Janeiro)

Ceres Alves de Araújo

(Pontifícia Universidade Católica de São Paulo)

Carlos Augusto de Medeiros

(Centro Universitário de Brasília)

Cícero Emidio Vaz

(Pontifícia Universidade Católica do Rio Grande do Sul)

Ciomara Ribeiro Silva Benincá

(Universidade de Passo Fundo)

Clara Maria Melo dos Santos

(Universidade Federal do Rio Grande do Norte)

Clara Virginia de Queiroz Pinheiro

(Universidade de Fortaleza)

Cláudio Vaz Torres

(Universidade de Brasília)

Cleci Maraschin

(Universidade Federal do Rio Grande do Sul)

Cleonice Pereira dos Santos Camino

(Universidade Federal da Paraíba)

Daniela Ribeiro Schneider

(Universidade Federal de Santa Catarina)

Débora Cristina Hipólide

(Universidade Federal de São Paulo)

Deborah Zacarias Guedes

(Universidade Federal de Uberlândia)

Denise de Souza Fleith

(Universidade de Brasília)

Denise Maurano Mello

(Universidade Federal de Juiz de Fora)

Denise Ruschel Bandeira

(Universidade Federal do Rio Grande do Sul )

Denize Rosana Rubano

(Pontifícia Universidade Católica de São Paulo)

Diana Lúcia Moura Pinho

(Universidade de Brasília)

Diana Tosello Laloni

(Pontifícia Universidade Católica de Campinas)

Domingos Sávio Coelho

(Universidade de Brasília)

Dora Selma Fix Ventura

(Universidade de São Paulo)

Dorian Mônica Arpini

(Universidade Federal de Santa Maria)

Dóris Lieth Nunes Peçanha

(Universidade Federal de São Carlos)

Edna Maria Marturano

(Universidade de São Paulo) 
Edna Maria Severino Peters Kahhale (Pontifícia Universidade Católica de São Paulo)

Edson Luiz André de Sousa (Universidade Federal do Rio Grande do Sul)

Eduardo Henrique Passos Pereira (Universidade Federal Fluminense)

Elaine Romero (Universidade Federal do Rio de Janeiro)

Eliane Mauerberg de Castro (Universidade Estadual Paulista Júlio de Mesquita Filho)

Elenice Seixas Hanna (Universidade de Brasília)

Eleonora Cavalcante (Universidade Estadual de Campinas)

Elisa Medici Pizao Yoshida (Pontifícia Universidade Católica de Campinas)

Eloiza da Silva Gomes de Oliveira (Centro Federal de Educação Tecnológica Celso Suckow da Fonseca)

Elza Maria do Socorro Dutra (Universidade Federal do Rio Grande do Norte)

Emerson Fernando Rasera (Universidade Federal de Uberlândia)

Emmanuel Zagury Tourinho (Universidade Federal da Paraíba)

Eulina da Rocha Lordelo (Universidade Federal da Bahia)

Eucia Beatriz Lopes Petean (Universidade de São Paulo)

Érika Arantes de Oliveira (Universidade de São Paulo - Ribeirão Preto)

Eveline Maria Leal Assmar (Universidade Salgado de Oliveira)

Evely Boruchovitch (Universidade Estadual de Campinas)

Francisco Javier Guerrero Ortega (Universidade do Estado do Rio de Janeiro)

Frederico Guilherme Graeff (Universidade de São Paulo)

Fúlvia Maria de Barros Mott Rosemberg (Fundação Carlos Chagas)

Gabriel Sperandio Milan (Universidade de Caxias do Sul)

Geraldina Porto Witter (Universidade Camilo Castelo Branco)

Gerson Américo Janczura (Universidade de Brasília)

Gérson Aparecido Yukio Tomanari (Universidade de São Paulo)

Gimol Benzaquen Perosa (Universidade Estadual Paulista Júlio de Mesquita Filho)

Gisele Maria Schwartz (Universidade Estadual Paulista Júlio de Mesquita Filho)

Gláuia Ribeiro Starling Diniz. (Universidade de Brasília)

Graciela Rene Ormezzano (Universidade de Passo Fundo)

Gustavo Adolfo Ramos Mello Neto (Universidade Estadual de Maringá)

Hartmut Günther (Universidade de Brasília)

Helena Maria Tannhauser Barros (Fundação Faculdade Federal de Ciências Médicas de Porto Alegre)

Henrique Soares Carneiro (Universidade de São Paulo)

Herculano Ricardo Campos (Universidade Federal do Rio Grande do Norte)

Idméa Semeghini-Siqueira (Universidade de São Paulo)
Irani Iracema de Lima Argimon

(Pontifícia Universidade Católica do Rio Grande do Sul)

Irai Cristina Boccato Alves

(Universidade de São Paulo)

Iray Carone

(Universidade Paulista)

Isabel Maria Farias Fernandes de Oliveira

(Universidade Federal do Rio Grande do Norte)

Isolda de Araújo Günther

(Universidade de Brasília)

Janice Inchauspe Pereira

(Pontifícia Universidade Católica do Rio Grande do Sul)

Jairo Eduardo Borges-Andrade

(Universidade de Brasília)

Jean Nunes dos Santos

(Universidade Federal da Bahia)

João dos Santos Carmo

(Universidade da Amazônia)

Jorge Mendes de Oliveira Castro Neto

(Universidade de Brasília)

Jorge Ponciano Ribeiro

(Universidade de Brasília)

José Antônio Damásio Abib

(Universidade Federal de São Carlos)

José Aparecido da Silva (Universidade de São Paulo)

José Augusto Evangelho Hernandez (Faculdades São Judas Tadeu)

José Carlos Fernandes Galduróz. (Universidade Federal de São Paulo)

José de Oliveira Siqueira

(Universidade de São Paulo)

Josele Regina de Oliveira Abreu Rodrigues

(Universidade de Brasília)

José Eduardo Pandossio (Universidade Católica de Brasília)

José Gonçalves Medeiros (Universidade Federal de Santa Catarina)

José Luiz Aidar Prado

(Pontifícia Universidade Católica de São Paulo)

José Mauro Gonçalves Nunes

(Universidade do Estado do Rio de Janeiro)

José Moysés Alves (Universidade Federal do Pará)

José Roberto da Silva Brêtas (Universidade Federal de São Paulo)

Julia Sursis Nobre Ferro Bucher

(Universidade de Fortaleza)

Junia de Vilhena

(Pontifícia Universidade Católica do Rio de Janeiro)

Katia Puente-Palacios

(Universidade de Brasília)

Kester Carrara

(Universidade Estadual Paulista Júlio de Mesquita Filho)

Laércia Abreu Vasconcelos

(Universidade de Brasília)

Leandro de Lajonquière

(Universidade de São Paulo)

Leandro Feitosa Andrade

(Fundação Carlos Chagas)

Leia Priszkulnik

(Universidade de São Paulo)

Leila Sanches de Almeida

(Universidade Federal do Rio de Janeiro)

Leila Strazza de Azevedo

(Universidade de São Paulo)

Leônia Cavalcante Teixeira

(Universidade de Fortaleza)

Liana Fortunato Costa

(Universidade de Brasília) 
Licia Barcelos de Souza

(Universidade de São Paulo)

Lilian Milnitsky Stein

(Pontifícia Universidade Católica do Rio Grande do Sul)

Lívia de Oliveira Borges

(Universidade Federal do Rio Grande do Norte)

Lucia de Mello e Souza Lehmann

(Universidade Federal do Estado do Rio de Janeiro)

Lucia Helena Tiosso Moretti

(Universidade Estadual de Londrina)

Luciana Mourão Cerqueira e Silva

(Universidade Salgado de Oliveira)

Luc Marcel Adhemar Vandenberghe

(Universidade Católica de Goiás)

Luis Felipe Rios do Nascimento

(Universidade Federal de Pernambuco)

Luis Nicolau Pares

(Universidade Federal da Bahia)

Luiz Carlos Couto de Albuquerque Moraes

(Universidade Federal de Minas Gerais)

Luiz Carlos de Oliveira

(Universidade do Sagrado Coração)

Maria Amália Pie Abib Andery

(Pontifícia Universidade Católica de São Paulo)

Magali Aparecida Alves de Moraes

(Faculdade de Medicina de Marília)

Mara Cristina Souza de Lucia

(Universidade de São Paulo)

Marcelo Quintino Galvão Baptista

(Universidade Federal do Pará)

Márcia Regina Marcondes Pedromônico

(Universidade Federal de São Paulo)

Márcia Siqueira de Andrade

(Centro Universitário Fieo)

Marcos Eduardo Scheicher

(Universidade Estadual Paulista Júlio de Mesquita Filho)

Marcus Túlio Caldas

(Universidade Católica de Pernambuco)

Maria Alves de Toledo Bruns

(Universidade de São Paulo - Ribeirão Preto)

Maria Aparecida Penso

(Universidade Católica de Brasília)

Maria Beatriz Martins Linhares

(Universidade de São Paulo)

Maria Claudia Santos Lopes de Oliveira

(Universidade de Brasília)

Maria da Consolação Gomes Cunha Fernandes Tavares

(Universidade Estadual de Campinas)

Maria da Graça Correa Jacques

(Universidade Federal do Rio Grande do Sul)

Maria da Penha de Lima Coutinho

(Universidade Federal da Paraíba)

Maria da Piedade Resende da Costa

(Universidade Federal de São Carlos)

Maria do Carmo Fernandes Martins

(Universidade Federal de Uberlândia)

Maria Fátima Olivier Sudbrack

(Universidade de Brasília)

Maria Helena Baptista Vilares Cordeiro

(Universidade do Vale do Itajaí)

Maria Helena Leite Hünziker

(Universidade de São Paulo)

Maria Inês Bacellar Monteiro

(Universidade Metodista de Piracicaba)

Maria Inês Gandolfo Conceição

(Universidade de Brasília)

Maria José Rizzi Henriques

(Universidade Estadual do Oeste do Paraná)

Maria Júlia Kovacs

(Universidade de São Paulo)
Maria Luiza Dias Garcia

(Instituto de Ensino Superior de Arujá)

Maria Luiza Furtado Kahl

(Universidade Federal de Santa Maria)

Maria Renata Machado Vaz Pinto Coelho

(Universidade Presbiteriana Mackenzie)

Maria Teresa de Silveira Pinheiro

(Universidade Federal do Rio de Janeiro)

Maria Therezinha de Lima Monteiro

(Universidade Católica de Brasília)

Marilda Emmanuel Novaes Lipp

(Pontifícia Universidade Católica de Campinas)

Marília Ferreira Dela Coleta

(Universidade Federal de Uberlândia)

Marília Marques da Silva

(Universidade Católica de Brasília)

Marilícia Witzler Antunes Ribeiro Palmieri

(Universidade Estadual de Londrina)

Marilza Terezinha Soares de Souza

(Universidade de Taubaté)

Mário César Ferreira

(Universidade de Brasília)

Marilda Emmanuel Novaes Lipp

(Pontifícia Universidade Católica de Campinas)

Marilene Aparecida Grandesso

(Pontifícia Universidade Católica de São Paulo)

Marina Pereira Gomes

(Universidade Católica de São Paulo)

Marina Pereira Rojas Boccalandro

(Pontifícia Universidade Católica de São Paulo)

Marisa Maria Brito da Justa Neves

(Universidade de Brasília)

Mathilde Neder

(Pontifícia Universidade Católica de São Paulo)

Mauricio Robayo Tamayo

(Universidade de Brasília)

Mercedes Villa Cupolillo

(Centro Universitário Estadual da Zona Oeste)

Meyre Eiras de Barros Pinto

(Universidade Estadual de Londrina)

Mirlene Maria Matias Siqueira

(Universidade Metodista de São Paulo)

Monah Winograd

(Pontifícia Universidade Católica do Rio de Janeiro)

Mylena Pinto Lima Ribeiro

(Centro Universitário de Vila Velha)

Nielsy Helena Puglia Bergamasco

(Universidade de São Paulo)

Nilson Gomes Vieira Filho

(Universidade Federal de Pernambuco)

Norberto Abreu de Silva Neto

(Universidade de Brasília)

Norberto Cysne Coimbra

(Universidade de São Paulo)

Odair Furtado

(Universidade de Brasília)

Olavo de Faria Galvão

(Universidade Federal da Paraíba)

Oswaldo Hajime Yamamoto

(Universidade Federal do Rio Grande do Norte)

Patrícia Waltz Schelini

(Universidade Federal de São Carlos)

Paulo Afrânio Sant'Anna

(Universidade Presbiteriana Mackenzie)

Paulo Albertini

(Universidade de São Paulo)

Paulo Rogério Meira Menandro

(Universidade Federal do Espírito Santo)

Raquel Maria de Melo

(Universidade de Brasília) 
Rachel Nunes da Cunha

(Universidade de Brasília)

Rafael Raffaelli

(Universidade Federal de Santa Catarina)

Raul Aragão Martins

(Universidade Estadual Paulista Júlio de Mesquita Filho)

Regina Aiko Fukunaga

(Pontifícia Universidade Católica de São Paulo)

Regina Helena Freitas Campos

(Universidade Federal de Minas Gerais)

Renata Ferrarez Fernandes Lopes

(Universidade Federal de Uberlândia)

Ricardo Ferreira Bento

(Universidade de São Paulo)

Ricardo Timm de Souza

(Pontifícia Universidade Católica do Rio Grande do Sul)

Richard Theisen Simanke

(Universidade Federal de São Carlos)

Rivadávio Fernandes Batista de Amorim

(Universidade Católica de Brasília)

Roberto Alves Banaco

(Pontifícia Universidade Católica de São Paulo)

Roberto Banaco

(Pontifícia Universidade Católica de São Paulo)

Roberto Novaes de Sá

(Universidade Federal Fluminense)

Rodrigo Sanches Peres

(Universidade de São Paulo)

Ronaldo Pilati

(Universidade de Brasília)

Ronald João Jacques Arendt

(Universidade do Estado do Rio de Janeiro)

Rosa Maria Stefanini de Macedo

(Pontifícia Universidade Católica de São Paulo)

Rosana de Fátima Possobon

(Universidade Estadual de Campinas)

Rosana Maria Tristão

(Universidade de Brasília)

Ruth Helena Pinto Cohen

(Universidade Federal do Rio de Janeiro)

Sadao Omote

(Universidade Estadual Paulista Júlio de Mesquita Filho)

Sandra Beatriz Chaves Tarquínio

(Universidade Federal de Pelotas)

Sandra Francesca Conte de Almeida

(Universidade Católica de Brasília)

Sebastião de Sousa Almeida

(Universidade de São Paulo)

Sergio Antonio da Silva Leite

(Universidade Estadual de Campinas)

Sergio Ozella

(Pontifícia Universidade Católica de São Paulo)

Silvia Helena Koller

(Universidade Federal do Rio Grande do Sul)

Silvia Marina Anaruma

(Universidade Estadual Paulista Júlio de Mesquita Filho)

Silvia Pereira da Cruz Benetti

(Universidade do Vale do Rio dos Sinos)

Sinesio Gomide Júnior

(Universidade Federal de Uberlândia)

Solange Jobim e Souza

(Universidade do Estado do Rio de Janeiro)

Solange Epelboim

(Universidade Estácio de Sá)

Solange Wechsler

(Pontifícia Universidade Católica de Campinas)

Sonia Grubits

(Universidade Católica Dom Bosco)
Sônia Maria Mello Neves

(Universidade Católica de Goiás)

Sonia Regina Loureiro

(Universidade de São Paulo)

Sônia Regina Fiorim Enumo

(Universidade Federal do Espírito Santo)

Sonia Regina Pasian

(Universidade de São Paulo)

Stela Miller

(Universidade Estadual Paulista Júlio de Mesquita Filho)

Sueli Aparecida Freire

(Universidade Federal de Uberlândia)

Suerde Miranda de Oliveira Brito

(Universidade Estadual da Paraíba)

Sylvia Beatriz Joffily

(Universidade Estadual do Norte Fluminense Darcy Ribeiro)

Tânia Cristina Rivera

(Universidade de Brasília)

Tânia Mara Campos de Almeida

(Universidade Católica de Brasília)

Tânia Mara Sperb

(Universidade Federal do Rio Grande do Sul)

Tânia Maria Cemin Wagner

(Universidade de Caxias do Sul)

Tereza Cristina Cavalcante Ferreira de Araújo

(Universidade de Brasília)

Terezinha Camargo de Viana

(Universidade de Brasília)

Terezinha Féres-Carneiro

(Pontifícia Universidade Católica do Rio de Janeiro)

Tereza Maria de Azevedo Pires Sério

(Pontifícia Universidade Católica de São Paulo)

Tufi Machado Soares

(Universidade Federal de Juiz de Fora)

Ulisses Ferreira de Araújo

(Universidade de São Paulo - Leste)

Valdiney Veloso Gouveia

(Universidade Federal da Paraíba)

Vera Decnop Coelho

(Universidade de Brasília)

Vera Lúcia de Mattos

(Universidade Estácio de Sá)

Vera Lucia Trindade Gomes

(Universidade do Estado do Rio de Janeiro)

Verônica Morais Ximenes

(Universidade Federal do Ceará)

Vicente de Paula Faleiros

(Universidade Católica de Brasília)

Violante Augusta Batista Braga

(Universidade Federal do Ceará)

Waldir Beividas

(Universidade Federal do Rio de Janeiro)

Walter Ernesto Ude Marques

(Universidade Federal de Minas Gerais)

Wânia Cristina de Souza

(Universidade de Brasília)

Wiliam Siqueira Peres

(Universidade Estadual Paulista Júlio de Mesquita Filho)

William Barbosa Gomes

(Universidade Federal do Rio Grande do Sul)

William Doise

(Université de Genève)

Zélia Maria de Melo

(Universidade Católica de Pernambuco)

Zilda Aparecida Pereira Del Prette

(Universidade Federal de São Carlos) 